\title{
Agujeros negros supermasivos
}

Maria Cecilia Tomasini

\section{Resumen}

Las observaciones del centro de la Vía Láctea y de otras galaxias parecen indicar que existe un agujero negro supermasivo en el centro de cada galaxia. Se ha encontrado que los movimientos de las estrellas del bulbo galáctico se encuentran vinculados a la masa del agujero negro supermasivo que reside en su centro. Estas relaciones indicarían que estos cuerpos superdensos se encuentran estrechamente ligados a las galaxias que los alojan y que habrían influido en su evolución. Si bien su origen y su formación son todavía un misterio su origen remoto podría asociarse a los quasars y explicaría su intensa luminosidad. En este trabajo revisaremos algunas características generales de los agujeros negros y nos referiremos brevemente a algunas de los descubrimientos recientes y a las futuras perspectivas de investigación.

Palabras clave: agujeros negros; galaxias; quasars; evolución estelar.

\section{Abstract}

Observations from the center of the Milky Way and other galaxies suggest that there is a supermassive black hole at the center of each galaxy. Strong relationships between the movements of the stars in the galactic bulge and the mass of the supermassive black hole that lies at its center have been found. These relationships indicate that these superdense bodies are closely linked to the galaxies that host them. Also, they probably have influenced their evolution. Although the origin and formation of supermassive black holes remain a mystery they could be associated with quasars and could explain its intense luminosity. In this paper we review some general characteristics of black holes and refer briefly to the recent research and future perspectives.

i Facultad de Ingeniería. Departamento de Cs. Exactas. Docente. Universidad de Palermo, Argentina. 


\section{Introducción}

Durante décadas se pensó que los agujeros negros eran construcciones matemáticas sin realidad física. Sin embargo la evidencia en favor de su entidad real es cada vez mayor. La astrofísica actual no sólo sostiene la existencia de los agujeros negros sino que supone que aparecen por doquier y a gran escala. Concretamente, se cree que cada una de las miles de galaxias que pueblan nuestro universo aloja un gigantesco agujero negro en su centro.

En un agujero negro la materia de una estrella de tamaño colosal ha colapsado en un volumen pequeñísimo. Por lo tanto su densidad es enorme. Efectivamente un agujero negro es un objeto tan compacto y denso que distorsiona profundamente el espacio y el tiempo a su alrededor. El campo gravitatorio de los agujeros negros es tan intenso que no permite que la radiación electromagnética - de la cual la luz visible forma parteescape de ellos. En consecuencia son invisibles. De allí su nombre. El agujero negro se encuentra rodeado de una superficie esférica llamada horizonte de los eventos. Éste puede ser atravesado en una única dirección: desde afuera hacia adentro. Las partículas y la luz pueden penetrarlo hacia el interior, pero no pueden escapar desde su interior hacia el universo exterior. Por lo tanto el horizonte de los eventos es un punto de no retorno.

Dado que todas las estrellas rotan lo más probable es que los agujeros negros mantengan su rotación luego de colapsar. Los agujeros negros rotantes atraen o acretan la materia de sus alrededores haciéndola rotar. La materia acretada se acumula por afuera del horizonte de los eventos formando un disco plano que se denomina disco de acreción. Cerca del horizonte, donde la gravedad es más intensa, la materia del disco es recalentada a varios cientos de miles de grados de temperatura e irradia intensamente en la región X del espectro electromagnético antes de caer definitivamente hacia el centro.

En la Teoría de la Relatividad General los agujeros negros rotantes están descriptos por la métrica de Kerr. La métrica de Schwarzschild es un caso particular de la métrica de Kerr, que describe agujeros negros sin rotación.

\section{Tipos de agujeros negros}

Los agujeros negros se clasifican en cuatro tipos según su masa. Los agujeros negros estelares se forman por colapso gravitacional en las etapas finales de vida de una estrella cuya masa supera los $3 \mathrm{M}_{\odot}$ donde el símbolo $\mathrm{M}_{\odot}$ indica una masa solar. ${ }^{1}$ Más adelante explicaremos el proceso por el cual una estrella podría colapsar en un agujero negro.

1 Denotamos la masa solar por el símbolo $\mathrm{M}_{\odot}$. Esta unidad de uso corriente en astrofísica equivale a la masa del Sol, que es $1,989 \times 10^{30} \mathrm{~kg}$. 
Los agujeros negros supermasivos son aquellos cuyas masas están comprendidas entre $10^{6}$ y $10^{9} \mathrm{M}_{\odot}$. Se cree que se alojan en el centro de las galaxias activas. Se denomina así a cierto tipo de galaxias que presentan una extraordinaria y violenta actividad impulsada por alguna fuente altamente energética localizada en sus núcleos. Las primeras galaxias activas que se descubrieron fueron los quasars ( quasi stellar radio source). Los quasars son sumamente luminosos. Su luminosidad es entre $100 \mathrm{y}$ 1000 veces superior a la de cualquier otra galaxia pero es emitida desde un volumen muy pequeño. Por lo tanto los quasars deben ser objetos sumamente compactos. La intensa luminosidad podría ser el resultado de la acreción de materia por parte de un agujero negro ubicado en el centro. La materia acretada irradiaría enormes cantidades de energía gravitacional a medida que cae hacia el centro. Para explicar semejante luminosidad el agujero negro central debe tener una masa del orden de $10^{6}-10^{9} \mathrm{M}_{\odot}$. Actualmente se cree que existen agujeros negros supermasivos no sólo en el centro de los quasars y de las galaxias activas sino en el centro de todas las galaxias.

Entre los agujeros negros estelares y los supermasivos se encontrarían los agujeros negros intermedios, cuya existencia aún se cuestiona (Pooley and Rappaport, 2006). Su masa estaría comprendida entre $10^{2}$ y $10^{5} \mathrm{M}_{\odot}$. Se han hallado indicios de esta clase de objetos en algunos cluster globulares. ${ }^{2}$ Por ejemplo, en el centro del cluster M15 que orbita alrededor de nuestra galaxia se ha hallado un cuerpo central cuya masa se ha estimado en $4000 \mathrm{M}_{\odot}$ (Gerssen et al., 2002). También se cree que existe un agujero negro intermedio de masa $2 \times 10^{4} \mathrm{M}_{\odot}$ en el centro del cluster G1 próximo a la galaxia de Andrómeda (Gebhardt et al., 2002).

Por último, se ha especulado acerca de la existencia de agujeros negros primordiales de masa muy inferior a $1 \mathrm{M}_{\odot}$. Este tipo de agujeros negros sólo pueden haberse generado en el universo temprano cuando la densidad de la materia era suficientemente alta. El estudio de este tipo de objetos requiere de las herramientas de la Teoría de Campos e incluso de la Gravedad Cuántica, teoría que aún no se ha desarrollado por completo.

\section{Cómo se forma un agujero negro estelar}

El agujero negro estelar es el producto final de la evolución de algunas de las estrellas de la secuencia principal. Al ordenar las estrellas en un diagrama de luminosidad versus temperatura superficial se observa que la distribución no es uniforme sino que las estrellas tienden a concentrarse en ciertas áreas. La secuencia principal es la banda central de esta distribución. Se la denomina de esta manera

2 Un cluster globular o cúmulo globular es un conjunto de $10^{5}-10^{6}$ estrellas que se encuentran gravitacionalmente ligadas formando un grupo compacto. Los cluster globulares se encuentran orbitando alrededor de las galaxias. Sus estrellas pertenecen a la población II, es decir que se trata de estrellas viejas. 
porque la mayor parte de las estrellas observadas se encuentran en esta franja. Las estrellas de la secuencia principal obtienen su energía y luminosidad transformando hidrógeno en helio mediante reacciones nucleares de fusión. Las estrellas de la secuencia principal mueren de diferente manera según cual sea su masa.

Mientras la estrella se mantiene en la secuencia principal sigue brillando gracias a las reacciones nucleares de fusión que se verifican en su núcleo. La presión de estas reacciones nucleares contrarresta a la fuerza gravitacional evitando el colapso de la materia hacia el centro. Pero cuando la estrella ha agotado todo su combustible su núcleo se convierte en una masa de ${ }^{56} \mathrm{Fe} o{ }^{62} \mathrm{Ni}$ y ya no se verifican más reacciones de fusión. Entonces cesa la presión de las reacciones nucleares y sobreviene el colapso estelar. ${ }^{3} \mathrm{Si}$ la masa de la estrella es menor a $1.4 \mathrm{M}_{\odot}$ colapsará formando una enana blanca. Este valor se conoce como límite de Chandrasekhar.

Las estrellas como nuestro Sol, con masas relativamente pequeñas, mueren como enanas blancas. En estas estrellas la materia se comprime hacia el centro aumentando su densidad a tal punto que los electrones se encuentran muy próximos entre sí. Debido al Principio de Exclusión de Pauli se verifica entre los electrones una intensa repulsión que se conoce como presión del gas degenerado de electrones. Esta presión detiene el colapso gravitacional. El tamaño final de una enana blanca es aproximadamente el de la Tierra. Su densidad es del orden de $10^{6} \mathrm{gr} / \mathrm{cm}^{3}$. Se trata de objetos muy densos y calientes pero a pesar de sus altas temperaturas, su luminosidad es baja debido a su pequeño tamaño. Estas estrellas han llegado al final de su evolución. Se enfrían lentamente hasta apagarse por completo y convertirse en cuerpos sólidos y fríos que no emiten luz.

Las estrellas de la secuencia principal cuya masa excede el límite de Chandrasekhar llegan al final de su vida de un modo diferente. Cuando una estrella de estas características consume todo su combustible nuclear, la energía liberada durante el colapso gravitatorio expulsa su envoltura superficial causando una explosión de supernova que deja como remanente una estrella de neutrones. Debido a su gran masa la materia colapsada en el interior de estas estrellas alcanza densidades muy superiores a las de una enana blanca. A estas densidades los neutrones, que son también fermiones, experimentan repulsiones mutuas debido al principio de Exclusión de Pauli. Esta presión, conocida como presión del gas degenerado de neutrones, detiene el colapso gravitacional. El radio de una estrella de neutrones es de unos $10 \mathrm{~km}$ y su densidad es del orden de $10^{15} \mathrm{gr} / \mathrm{cm}^{3}$. Aunque no se conoce la ecuación de estado de una estrella de neutrones, se sabe con certeza que su masa no puede exceder las $3 \mathrm{M}_{\odot}$. Por encima de este valor, conocido como límite de Oppenheimer-Volkoff, la estrella colapsará como un agujero negro.

3 El proceso que se verifica en el interior de las estrellas es mucho más complejo. Su descripción excede los objetivos de este trabajo. Véase una explicación más detallada en E. Battaner, 1999; y en R. Kippenhahn and A. Weigert, 1989. 
Se cree que cuando la masa de una estrella de la secuencia principal excede las $3 \mathrm{M}_{\odot}$ ésta colapsará formando un agujero negro en un evento extremadamente luminoso, conocido como explosión de hipernova. Este evento se acompaña de abundante emisión de radiación gama. Estas emisiones han sido detectadas desde hace varias décadas y recientemente se las ha asociado a la formación de agujeros negros estelares (Atteia, 2012). En un agujero negro nada detiene al colapso gravitatorio. Por lo tanto la masa de la estrella se comprime alcanzando volúmenes ínfimos y densidades enormes.

\section{Detección de agujeros negros}

La forma habitual de observar el universo es a través de las radiaciones emitidas por los objetos astrofísicos, en diferentes regiones del espectro electromagnético. En la actualidad existen poderosos telescopios, muchos de ellos orbitando alrededor de la Tierra, que detectan emisiones de diferentes longitudes de onda. Por ejemplo, el Hubble es sensible en la región visible y en las regiones infrarroja y ultravioleta mientras que el Chandra lo es en la región de rayos X. Sin embargo los agujeros negros no emiten ningún tipo de radiación. Por lo tanto, hasta el momento presente, la única forma de inferir su presencia es a través de los efectos de su campo gravitatorio.

La primera evidencia de la existencia de agujeros negros se obtuvo a partir de la observación de sistemas estelares binarios. Un sistema binario es un conjunto de dos estrellas que orbitan una alrededor de la otra. En muchos sistemas binarios sólo una de las dos estrellas es visible. Se la observa rotando alrededor de un punto en el cual no aparece ningún astro visible. Sin embargo, en ese punto existe algún cuerpo que genera el campo gravitatorio suficiente como para obligar a la compañera visible a describir órbitas a su alrededor. Midiendo ciertos parámetros de la órbita de la estrella visible es posible estimar la masa su acompañante invisible. ${ }^{4}$ En algunos casos se ha encontrado que la masa del objeto invisible supera las $3 \mathrm{M}_{\odot}$. La hipótesis más razonable es suponer que el acompañante oscuro es un agujero negro.

El primer agujero negro detectado aplicando este método fue el ubicado en

4 Para estimar la masa del objeto invisible acompañante se necesitan varios parámetros relacionados con la estrella visible. Es necesario conocer su período de rotación y su velocidad en la dirección de la visual. Ambos parámetros pueden ser determinados a partir del corrimiento Doppler de sus líneas espectrales. También es necesario conocer la inclinación de su órbita respecto de la dirección de la visual. La imposibilidad de calcular con precisión este dato suele introducir grandes incertidumbres en el resultado final. También se necesita estimar su masa. Esto puede hacerse por diferentes métodos como por ejemplo su ubicación en un diagrama de luminosidad versus temperatura o diagrama de Hertzsprung - Russell. En algunos casos particulares puede estimarse su masa a partir de otros parámetros de su órbita como el semieje mayor y el período. 
Cygnus $\mathrm{X}-1$. Fue descubierto en 1965 y se encuentra a $2.5 \mathrm{kpc}$ de nosotros. ${ }^{5} \mathrm{Su}$ masa se ha estimado entre 6 y $20 \mathrm{M}_{\odot}$. Si bien la incerteza en la determinación de la masa es grande, todas las medidas efectuadas indican que el ente oscuro de ese sistema binario tiene una masa superior a $3 \mathrm{M}_{\odot}$ por lo que muy probablemente se trata de un agujero negro estelar. ${ }^{6}$ Luego del hallazgo de Cygnus $\mathrm{X}-1$ se han descubierto decenas de candidatos a agujeros negros estelares. Por ejemplo en la galaxia M 33 se ha detectado un sistema binario en el cual se han podido determinar con bastante precisión los parámetros, dando por resultado para el acompañante oscuro una masa de aproximadamente $15 \mathrm{M}_{\odot}$ (Orosz et al., 2007; Valsecchi et al., 2009; Valsecchi et al., 2011).

La observación de los centros de galaxias activas y del centro de nuestra propia galaxia proveen evidencia adicional en favor de la existencia de los agujeros negros. Los movimientos de las estrellas próximas al centro de la Vía Láctea y la intensa emisión de radiación desde algunos núcleos galácticos señalarían la presencia de objetos muy compactos en el centro de las galaxias. Las masas estimadas en estos casos oscilan ente 106 y $1010 \mathrm{M}_{\odot}$. Se cree que existe uno de estos agujeros negros en el centro de cada galaxia, incluida la nuestra. En virtud de las últimas evidencias se cree que existen también agujeros negros de masa intermedia en el centro de los cluster globulares (Gerssen et al, 2002. Gebhardt et al., 2002).

Otra forma de inferir la presencia de un agujero negro es mediante sus efectos como lente gravitacional. Un objeto compacto curva el espacio tiempo a su alrededor de tal manera que un haz de luz que pase cerca será desviado de su dirección original. Por lo tanto los cuerpos compactos actúan como lentes, causando la deflexión de la luz. El ángulo de deflexión está relacionado con la masa del objeto que lo causa. En consecuencia, midiendo el ángulo de deflexión es posible estimar esta la masa (Bartelmann and Schneider, 2001). Si la luz es deflectada por un ente invisible cuya masa es superior a $3 \mathrm{M}_{\odot}$ entonces podría inferirse la presencia de un agujero negro (Eiroa, 2012).

La condición de que la masa sea mayor a $3 \mathrm{M}_{\odot}$ es necesaria pero no suficiente para determinar que un objeto es un agujero negro. La única forma de probar que se trata efectivamente de un agujero negro es verificar que posee un horizonte de los eventos ya que ésta es la única característica que lo diferencia de otro tipo de estrella muy masiva y superdensa. Como hemos dicho anteriormente, cuando un agujero negro se encuentra ante un astro muy luminoso la luz que llega al observador es deflectada. Pero parte de los fotones emitidos por la fuente-aquellos que pasan más cerca- caen hacia el agujero negro generando una zona oscura

5 El parsec, abreviado como pc, es una unidad corrientemente utilizada en astronomía. Un pc equivale a $3.12 \times 10^{13} \mathrm{~km}$.

6 Las incertezas en las estimaciones proviene fundamentalmente del hecho de que se desconoce cuál es la inclinación de la órbita de la estrella visible respecto de la visual. Véase la nota 4. 
llamada sombra. La forma aparente del agujero negro es definida por la sombra. La observación directa de esta forma aparente es una de las mayores expectativas de la astrofísica puesto que proporcionará una descripción de la región próxima al horizonte de los eventos. En un futuro no muy lejano los proyectos RadioAstron en la región de radioondas y Maxim (Micro Arc second X-ray Imaging Mission) en la región $\mathrm{X}$ del espectro serán capaces de explorar el centro galáctico con gran resolución. Estos instrumentos realizarán mediciones de gran precisión en torno al agujero negro supermasivo que se localiza en el centro de nuestra Vía Láctea y se espera que puedan arrojar información acerca de su sombra y de su forma aparente (Eiroa, 2012).

\section{Agujeros negros supermasivos}

La evidencia en favor de la existencia de los agujeros negros supermasivos es cada vez mayor. Los modernos telescopios han permitido realizar mediciones en los centros galácticos que corroborarían la hipótesis de que existe un agujero negro no sólo en el centro de las galaxias activas sino en el centro de todas las galaxias. Se cree incluso que los agujeros negros supermasivos serían cruciales en el proceso de formación de las galaxias.

En el año 1974 se detectó una poderosa radiofuente en dirección al centro de nuestra Vía Láctea. Este hallazgo fue confirmado por posteriores investigaciones. Actualmente esta radiofuente recibe el nombre de Sagitario $A^{*}{ }^{-o}$ en forma abreviada SagA*-debido a que se ubica en la constelación de Sagitario. También se ha verificado que en las regiones centrales de nuestra galaxia hay un conjunto de estrellas cuyos movimientos han sido monitoreados con cuidado en los últimos años. Los resultados de estas observaciones han demostrado que las estrellas se mueven alrededor de un objeto invisible y muy compacto localizado exactamente donde se encuentra SagA*. Las órbitas de estas estrellas son elípticas y se ajustan a las Leyes de Kepler que describen el movimiento de los cuerpos celestes. Como el centro de nuestra galaxia está rodeado de polvo cósmico no puede ser observado con telescopios ordinarios ya que el polvo es opaco a las longitudes de onda de la región visible del espectro. Para escrutar el centro de nuestra galaxia fue necesario utilizar telescopios sensibles a las longitudes de onda de la franja infrarroja que pueden atravesar las nubes de polvo. Gracias a esta tecnología pudieron determinarse los parámetros orbitales de las estrellas que se mueven en torno a SagA*. Estos datos permitieron estimar la masa de la radiofuente en unas $4 \times 10^{6} \mathrm{M}_{\odot}$. Por tratarse de un cuerpo oscuro y muy masivo la hipótesis más razonable para explicar su naturaleza es que se trata de un gigantesco agujero 
negro (Ghez et al., 2008; Genzel et al., 2010).

Las observaciones de los centros de otras galaxias no son tan detalladas como las de la Vía Láctea debido a que se encuentran a grandes distancias. Sin embargo también en estos casos se ha hallado que la materia de las regiones centrales orbita rápidamente alrededor de algún objeto muy compacto. En muchos casos se verifica una enorme emisión de energía desde un volumen central muy pequeño. El único mecanismo que puede explicar razonablemente semejante emisión de energía desde una región tan reducida es la acreción de materia por parte de un gigantesco agujero negro central. (Mc Connell et al., 2011; Pastorini et al., 2008).

El proceso mediante el cual se forman los agujeros negros supermasivos en el centro de las galaxias se desconoce pero se supone que se generan en las primeras etapas de formación de las mismas y que se asocian a su evolución (Cattaneo et al., 2009). Midiendo diversas galaxias se ha encontrado que la masa del objeto central se relaciona con la masa del bulbo -o protuberancia- según la relación $\mathrm{M}_{\mathrm{BH}}$ $\sim 0.006 \mathrm{M}_{\text {BULBO }}$ (Magorrian et al., 1998). ${ }^{8}$ Esta relación permitiría suponer que en el momento de formación de las galaxias aproximadamente el 0.6 por ciento de la materia del bulbo colapsa hacia el centro formando un agujero negro supermasivo. El gas acretado por el agujero negro en esta etapa irradiaría enormes cantidades de energía generando un quasar. Posteriormente el agujero negro central dejaría de acretar materia a esas tasas y se convertiría en un agujero negro inactivo. Esos agujeros negros conformarían los centros de las galaxias actuales. En otras palabras, es posible que toda galaxia presente haya sido alguna vez un quasar aunque los mecanismos que gobiernan estos cambios no se conocen por completo.

La inspección de distintas galaxias ha permitido inferir otra importante relación entre la masa MBH del agujero negro y la dispersión $\sigma$ en las velocidades de las estrellas de la galaxia que lo contiene. Esta relación es otro indicio de que existe algún vínculo causal entre la formación y la evolución del agujero negro y del bulbo de la galaxia. Todo parece señalar que el bulbo y el agujero negro central se forman y se desarrollan como parte de un mismo proceso. Dado que la relación $\mathrm{M}_{\mathrm{BH}}{ }^{-} \sigma$ se verifica incluso para estrellas alejadas del centro galáctico su determinación constituye un método inapreciable para estudiar galaxias distantes en las cuales se requerirían altísimas resoluciones para observar el centro (Gebhardt et al., 2000; Ferrarese and Merritt, 2000; Merritt and Ferrarese, 2001).

7 Se han realizado también observaciones del disco maser de agua que rodea el núcleo de ciertas galaxias como la NGC 4258 permitiendo estimar una masa para el objeto central del orden de $10^{7} \mathrm{M}_{\odot}$ (Siopis et al, 2009). Un maser astrofísico es un amplificador natural de ondas en el rango de las microondas. En algunos casos su composición es rica en moléculas de agua por lo que se los denomina masers de agua.

8 Una galaxia espiral está formada por el bulbo o protuberancia, el disco y el halo. El bulbo galáctico es el conjunto de estrellas que se encuentran en la región central. 
Otra de las hipótesis que intenta explicar el proceso que conduce a la formación de agujeros negros supermasivos en el centro de las galaxias sugiere que estos gigantescos objetos son fruto de la fusión de agujeros negros más pequeños. La astrofísica actual apoya la idea de que las galaxias se fusionan entre sí formando galaxias de mayor tamaño (C. Baugh, 2006; Springer et al., 2005; Cole et al., 2000). Si esta hipótesis es cierta, probablemente los enormes agujeros negros centrales de las actuales galaxias se habrían formado a partir de la fusión de los agujeros negros alojados en galaxias más pequeñas. ${ }^{9}$ Esta última cuestión podría ser esclarecida si se detectan ondas gravitacionales en el marco del proyecto LISA (Laser Interferometer Space Antenna) ya que, según los modelos astrofísicos y relativistas, la fusión de galaxias y de agujeros negros iría acompañada de un intensa emisión de ondas gravitacionales. ${ }^{10}$

\section{Conclusión}

Los agujeros negros han sido considerados durante décadas como entes matemáticos puramente abstractos y dignos de la ciencia ficción. Sin embargo la astrofísica contemporánea está cada vez más convencida de su realidad física. Las últimas investigaciones señalan casi con certeza que los agujeros negros no sólo existen sino que muchos de ellos son supermasivos y rigen la dinámica y el destino de todas las galaxias. Esperamos que los desarrollos teóricos y tecnológicos de los próximos años nos permitan conocer a estos extraños cuerpos celestes con mayor profundidad.

9 El observatorio de rayos $X$ Chandra ha encontrado dos agujeros negros supermasivos orbitando uno alrededor del otro en la galaxia NGC 6240. Se cree que esta galaxia se ha formado recientemente a partir de la fusión de dos galaxias más pequeñas. Si cada una de ellas contenía un agujero negro central, esto podría explicar la presencia de dos agujeros negros muy próximos al centro en una única galaxia.

10 Técnicamente una onda gravitacional es una perturbación en la métrica del espacio tiempo; puede entenderse intuitivamente como una ondulación que se propaga a la velocidad de la luz en la trama espacio temporal. 


\section{Bibliografía}

Atteia, J. L. (2012). Gamma-Ray Bursts, witnessing the birth of stellar mass black holes. Proceedings of Science, 116.

Bartelmann, M and Schneider, P. (2001). Weak Gravitational Lensing. Physics Reports, 340, $291-472$.

Battaner, E. (1999). Introducción a la astrofisica. Madrid: Alianza.

Baugh, C. (2006). A primer on hierarchical galaxy formation: the semi-analytical approach. Rept. Prog. Phys., 69, 3101-3156.

Cattaneo, A; Faber, S. M.; Binney, J.; Dekel, A.; Kormendy, J.; Mushotzky, R. et al. (2009). The role of black holes in galaxy formation and evolution. Nature, $460,213-219$.

Cole, S.; Lacey, C.; Baught, C. and Frenk, C. (2000). Hierarchical Galaxy Formation. MNRA, 319, 168 - 208.

Eiroa, E. (2013). Strong deflection gravitational lensing. Gravitation, Relativistic Astrophysics and Cosmology, En Proceedings of the First ArgentinianBrazilian Meeting, (33-52). Sao Paulo: Livraria da Fisica.

Ferrarese, L. and Merritt, D. (2000). A fundamental relation between supermassive black holes and their host galaxies. Astrophys. J., 539, 9-12. (2002). Supermassive black holes. Physics World, june 2002, 1-6.

Gebhardt, K; Rich, R. M. and Ho, L. C. (2002). A 20 thousand solar mass black hole in the stellar cluster G1. Astrophys. J., 578, $41-46$.

Gebhardt, K.; Bender, R.; Bower, G.; Dressler, A.; Faber, S. M.; Filippenko, A. V. et al. (2000). A relationship between nuclear black hole mass and galaxy velocity dispersion. Astrophys. J., 539, 13-16.

Genzel, R.; Eisenhauer, F. and Gillessen, S. (2010) The Galactic Center massive black hole and nuclear star cluster. Rev. Mod. Phys., 82, 3121-3258.

Gerssen, J.; van der Marel, R. P.; Gebhardt, K.; Guhathakurta, P; Peterson, R. C. and Pryor, C. (2002). Hubble Space Telescope Evidence for an IntermediateMass Black Hole in the Globular Cluster M15-II. Kinematical Analysis and Dynamical Modeling. Astron. J., 124, 3270-3313

Ghez, A. M.; S. Salim, S.; Weinberg, N. N.; Lu, J. R.; Do, T.; Dunn, J. K. et 
al. (2008). Measuring distance and properties of the Milky Way's central supermassive black hole with stellar orbits. Astrophys. J., 689, $1044-1062$.

Hobson, M. P.; Efstathiou, G. P. and Lasenby, A. N. (2006). General Relativity. An Introduction for Physicists. New York: Cambridge University Press.

King, A. (2003). Black holes, galaxy formation, and the MBH- $\sigma$ relation. Astrophys. J., 596, 27-29.

Kippenhahn, R. and Weigert, A. (1989). Stellar Structure and Evolution. Munich: Springer - Verlag.

Magorrian, J.; Tremaine, S.; Richstone, D.; Bender, R.; Bower, G.; Dressler, A. et al. (1998). The demography of massive dark objects in galaxy centers. Astron. J., 115, 2285 - 2305.

Marconi, A. and Hunt, L. (2003). The relation between black hole mass, bulge mass, and near-infrared luminosity. Astrophys. J., 589, 21-24.

McConnell, N. J.; Ma, C.; Gebhardt, K.; Wright, S. A.; Murphy, J. D.; Lauer, T. R. et al. (2011). Two ten-billion-solar-mass black holes at the centres of giant elliptical galaxies. Nature, 480, 215-218.

Merritt, D. and Ferrarese, L. (2001). Relationship of Black Holes to Bulges. En The Central kpc of Starbursts and AGN. ASP Conference Series, xxx, $335-$ 363.

Orosz, J. A.; McClintock, J. E.; Narayan, R.; Bailyn, C. D.; Hartman, J. D.; Macri, L. et al. (2007). A 15.56 solar mass black hole in an eclipsing binary in the nearby spiral galaxy M 33. Nature, 449, $872-875$.

Pastorini, G.; Marconi, A.; Capetti, A.; Axon, A. J.; Alonso-Herrero, A.; Atkinson, J. et al. (2006). Supermassive black holes in the Sbc spiral galaxies NGC 3310, NGC 4303 and NGC 4258. Astronomy and astrophysics, 469, 2, 405-423.

Pooley, D. and Rappaport, S. (2006). X-Rays from the Globular Cluster G1: intermediate mass black hole or low mass X-Ray binary?. Astrophys. J., 644, $45-48$.

D. Raine and E. Thomas, Black Holes. An Introduction. Imperial College Press, 2010 .

Siopis, C.; Gebhardt, K.; Lauer, T. R.; Kormendy, J.; Pinkney, J.; D. Richstone, 
D. et al. (2009).A Stellar Dynamical Measurement of the Black Hole Mass in the Maser Galaxy NGC 4258. Astrophys. J. 693, $946-969$.

Springel, V.; White, S. D. M.; Jenkins, A.; Frenk, C. S.; Yoshida, N.; Gao, L. et al. (2005). Simulations of the formation, evolution and clustering of galaxies and quasars. Nature, 435, $629-636$.

Valsecchi, F.; Glebbeek, E.; Farr, W. M.; Fragos, T.; Willems, B.; J. A. Orosz, J. A. et al. (2011). An Evolutionary Model for the Massive Black Hole X-ray Binary M33 X-7. En Evolution of compact binaries. ASP Conference Series, $447,271-277$.

Valsecchi, F.; Willems, B.; Fragos, T. and Kalogera, V.(2009). The Eclipsing Black Hole X-ray Binary M33 X-7: Understanding the Current Properties. En Hot And Cool: Bridging Gaps in Massive Star Evolution. ASP Conference Series, XXX.

Van der Marel, R. (2012). The Milky Way's Collision with the Andromeda Galaxy. Hubble. Science year in review, $65-75$.

Wang, Q. D. and Stolovy, S. (2009). The center of the Milky Way Galaxy. Hubble. Science year in review, $89-100$. 